\title{
Maximum Obtainable Energy Harvesting Power from Galloping-Based Piezoelectrics
}

\author{
Mohammad Yaghoub Abdollahzadeh Jamalabadi $\left(\mathbb{D},{ }^{1,2}\right.$ Mostafa Safdari Shadloo, ${ }^{3}$ \\ and Arash Karimipour ${ }^{4}{ }^{4}$ \\ ${ }^{1}$ Department for Management of Science and Technology Development, Ton Duc Thang University, \\ Ho Chi Minh City 700000, Vietnam \\ ${ }^{2}$ Faculty of Civil Engineering, Ton Duc Thang University, Ho Chi Minh City 700000, Vietnam \\ ${ }^{3}$ CORIA-UMR 6614, Normandie University, CNRS-University \& INSA, 76000 Rouen, France \\ ${ }^{4}$ Dipartimento di Ingegneria Astronautica, Elettrica ed Energetica, Sapienza Universitá di Roma, Via Eudossiana 18, \\ Roma 00184, Italy
}

Correspondence should be addressed to Arash Karimipour; arashkarimipour@gmail.com

Received 4 March 2020; Accepted 11 May 2020; Published 7 September 2020

Academic Editor: Francesco Franco

Copyright ( $) 2020$ Mohammad Yaghoub Abdollahzadeh Jamalabadi et al. This is an open access article distributed under the Creative Commons Attribution License, which permits unrestricted use, distribution, and reproduction in any medium, provided the original work is properly cited.

\begin{abstract}
In this paper, the maximum obtainable energy from a galloping cantilever beam is found. The system consists of a bluff body in front of wind which was mounted on a cantilever beam and supported by piezoelectric sheets. Wind energy caused the transverse vibration of the beam and the mechanical energy of vibration is transferred to electrical charge by use of piezoelectric transducer. The nonlinear motion of the Euler-Bernoulli beam and conservation of electrical energy is modeled by lumped ordinary differential equations. The wind forces on the bluff body are modeled by quasisteady aeroelasticity approximation where the fluid and solid corresponding dynamics are disconnected in time scales. The linearized motion of beam is limited by its yield stress which causes to find a limit on energy harvesting of the system. The theory founded is used to check the validity of previous results of researchers for the effect of wind speed, tip cross-section geometry, and electrical load resistance on onset speed to galloping, tip displacement, and harvested power. Finally, maximum obtainable average power in a standard RC circuit as a function of deflection limit and synchronized charge extraction is obtained.
\end{abstract}

\section{Introduction}

Piezoelectric energy harvester uses the ambient energy and transfers it into electric charge [1-7]. The parametric study and the design of piezoelectric energy harvesting from galloping motion is studied by Barrero-Gil et al. [1]. First, experimental results are obtained by Sirohi and Mahadik [2, 3] using a cantilever beam exposed to air with constant velocity in a wind tunnel. Simulation of galloping cantilever coupled with a piezoelectric transducer in an electric circuit is performed by Abdelkefi et al. [4]. Analytical solution of that system of equations is presented in the work of Tan and Yan [5]. As the values for the harvested power of Abdelkefi et al. [4] and Tan and Yan [5] in some figures (watts) are beyond the order of the magnitude of experimental data of Sirohi and Mahadik [2, 3] and Jamalabadi et al. $[6,7]$ (milliwatts), this paper addressed the problem to the linear assumption of force-deflection relation for the Euler-Bernoulli beam. This research proposes to consider the limitation of the yield stress of piezoelectric material as the maximum point of mechanical stability as well as energy harvesting.

\section{Mathematical Model}

The schematic of the system is shown in Figure 1. A bluff body exposed to the free stream is mounted on an Euler-Bernoulli cantilever beam. The two piezoelectric wafers are attached on free surfaces of beam which are in an electric circuit with electric impedance. The $y$-direction galloping of the bluff body in the first mode of the structure is modeled by Abdelkefi et al. [4] by 


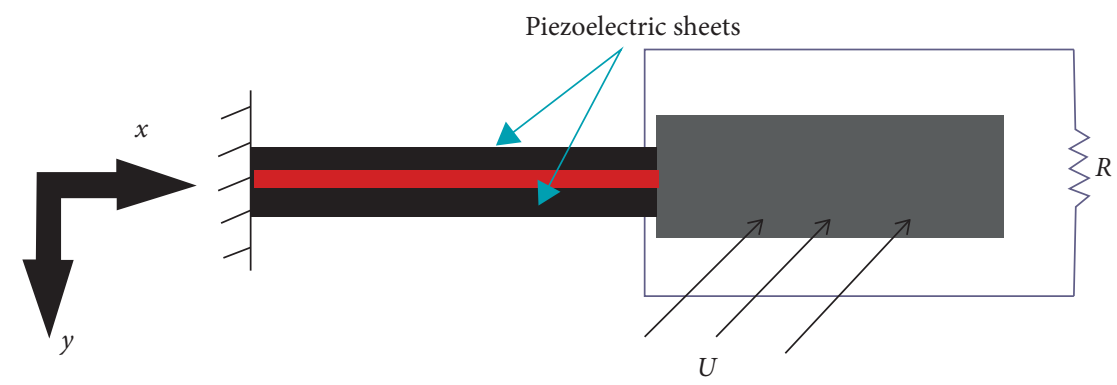

Figure 1: Schematic of the galloping piezoelectric energy harvester for the case of wind direction normal to the cantilever beam.

$$
\begin{array}{rlrl}
\ddot{y}(t)+(2 \xi \omega-A) \dot{y}(t)-B \phi(L)^{2} \dot{y}(t)^{3}+\omega^{2} y(t)+\phi(L) \theta_{p} V(t)=0, & (1) & V(t) & =\phi(L) y_{\max } \sin (\Omega t), \\
& V(t)=\sqrt{\mathrm{RC}} \Omega \phi(L) y_{\max } \sin (\Omega t+\alpha) .
\end{array}
$$

$$
\dot{V}(t)=-\frac{V(t)}{\mathrm{RC}_{p}}+\frac{\theta_{p}}{\phi(L) C_{p}} \dot{y}(t)
$$

The integration of equations (1) and (2) term for half period of motion, the onset of galloping, the maximum deflection of the beam, and power harvesting which are

By assuming the following functions for motion and voltage,

$$
\begin{aligned}
& U_{\text {min }}=\frac{2(2 \xi \omega+C)}{\rho_{\text {air }} b_{\text {tip }} a_{1}\left(\phi^{2}(L) L_{\text {tip }}+\phi(L) \phi^{\prime}(L) L_{\text {tip }}^{2}+1 / 3 \phi^{\prime 2}(L) L_{\text {tip }}^{3}\right)}, \\
& A_{\max }=\frac{\phi(L)}{\Omega} \sqrt{\frac{(C+2 \xi \omega-A)}{0.75 B}}, \\
& P_{\max }=\frac{C(2 \xi \omega+C-A)}{0.75 B} .
\end{aligned}
$$

Before going further to derive the optimal values of the system parameters, same as of Tan and Yan [5], it should be noticed that the maximum bending moment at the base of the beam is calculated by

$$
M_{\text {base }}(\dot{y})=\frac{(2 / 3) w_{\mathrm{PZT}}\left[\left(\left(t_{b} / 2\right)+t_{\mathrm{PZT}}\right)^{3}-t_{\mathrm{PZT}}^{3}\right]+(1 / 12) w_{b} t_{b}^{3}}{\left(\left(t_{b} / 2\right)+t_{\mathrm{PZT}}\right)} \sigma_{Y}
$$

where the yielding stress of piezoelectric material is about 31.2 $\mathrm{MPa}$ in the experiment.

\section{Results}

The numerical (solving equations (1) and (2)) and analytical (equations (5) and (6)) solutions are calculated based on the data provided [4]. The change in the amplitudes of the displacement of the tip of the cantilever beam with the free stream velocities at different electrical impedances from the numerical and analytical solutions are shown in Figure 2. As shown in
Figure 2, the analytical and numerical solutions are in a good agreement. In addition, the limitation considered in equation (7) affected the results, where soon after the onset of galloping, the system experienced the tear in the piezoelectric sheet. To better assess the consequences of the constraint of equation (7), the vertical axis of Figure 2 is plotted in a log scale. As shown, the maximum displacement that the beam can bear is less than the order of centimeter, and for such stiffness, the order of the deflection before failure is millimeter.

The variation in the harvested power with the electrical impedances at different free stream velocities from the 


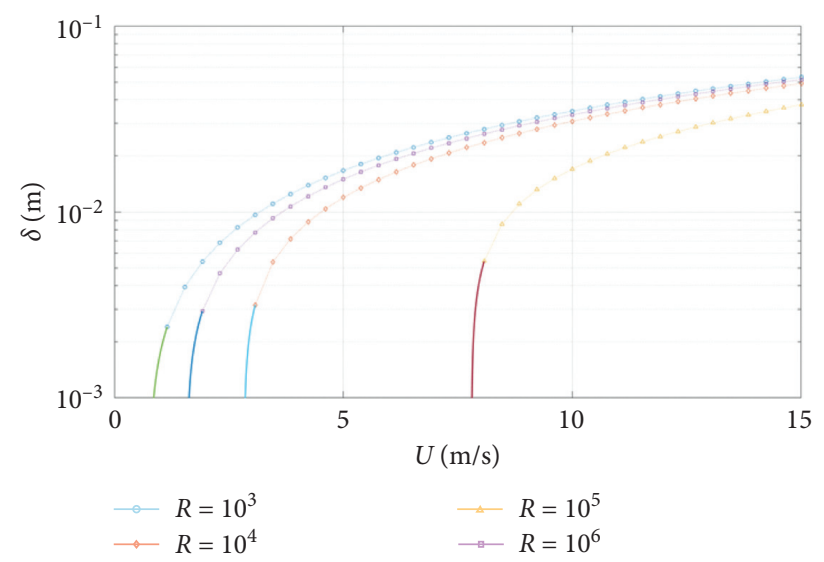

Figure 2: Analytical solutions (dash lines), numerical solutions (symbols), and corrected solutions (lines) of the amplitudes of the tip displacement versus the electrical impedance and free stream velocity.

analytical and numerical solutions is revealed in Figure 3. As shown in Figure 3, the results of numerical and analytical methods are in a good agreement. Additionally, the limitation considered in equation (7) affected the results, where soon after the onset of galloping, the system experienced the tear in the piezoelectric wafers and the harvesting of the wind energy is stopped. To see the significances of the constraint of equation (7) on the system clearer, the vertical axis in Figure 3 is schemed in a log scale. As exposed, the maximum harvested power in the electric circuit is less than the order of $10^{-1}$ watts, and for other cases, the order of the harvested power before failure is milliwatts. The results are in a good agreement with the experimental results $[2,3,6,7]$.

By differentiating equation (6) with respect to the parameter $C$, the optimal design of the electric circuit for the galloping system is obtained as $\left(\partial P_{\max } / \partial C=0 \Longrightarrow C_{o}=\right.$ $(A-2 \xi \omega / 2))$

$$
\begin{aligned}
R_{o} & =\frac{\theta_{p}^{2}+C_{p}(A-2 \xi \omega / 2)^{2} \pm \sqrt{\left(\theta_{p}^{2}+C_{p}(A-2 \xi \omega / 2)^{2}\right)^{2}-4 C_{p}(A-2 \xi \omega / 2)^{2}\left(C_{p} \omega^{2}+\theta_{p}^{2}\right)}}{C_{p}(A-2 \xi \omega)\left(C_{p} \omega^{2}+\theta_{p}^{2}\right)}, \\
U_{o}^{\text {onset }} & =\frac{4 \xi \omega}{\rho_{\text {air }} b_{\text {tip }} a_{1}} \frac{1}{\phi^{2}(L) L_{\text {tip }}+\phi(L) \phi^{\prime}(L) L_{\text {tip }}^{2}+1 / 3 \phi^{\prime 2}(L) L_{\text {tip }}^{3}}, \\
A_{\max , o} & =\phi(L) \sqrt{\frac{2 \xi \omega-A}{1.5 B \Omega^{2}}}, \\
P_{\max , o} & =-\frac{(2 \xi \omega-A)^{2}}{3 B}, \\
\Omega & =\omega \sqrt{\frac{1}{1-0.5 R_{\mathrm{co}} C_{p}(A-2 \xi \omega)}} .
\end{aligned}
$$

Analytical solutions, numerical solutions, and corrected solutions of the amplitudes of the harvested power versus the parameter $C$ and free stream velocity are plotted in Figure 4. As shown, again the limited range of parameter $C$ is allowed and the maximum obtainable power should be searched through those values.

When the value of expression under the square root in eqution (8) for $R_{o}$ is negative (the velocities higher than 


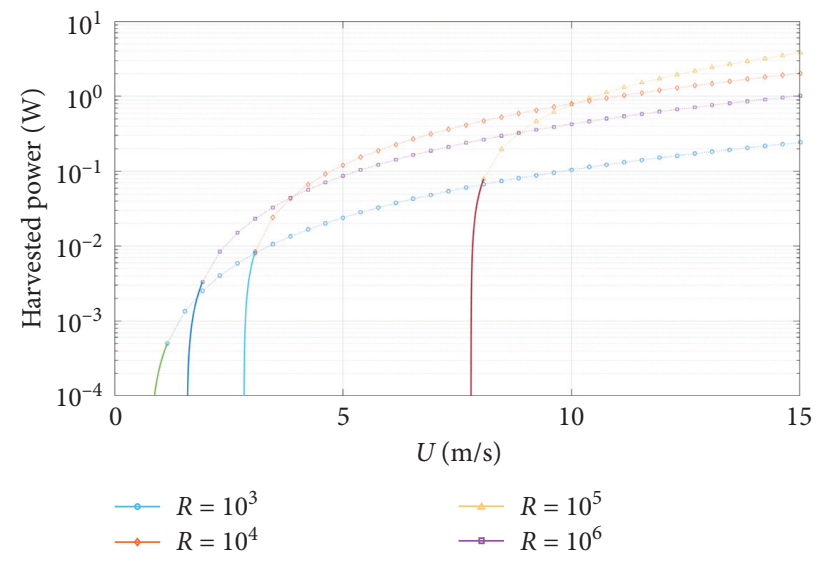

FIgURE 3: Analytical solutions (dash lines), numerical solutions (symbols), and corrected solutions (lines) of the amplitudes of the harvested power versus the electrical impedance and free stream velocity.

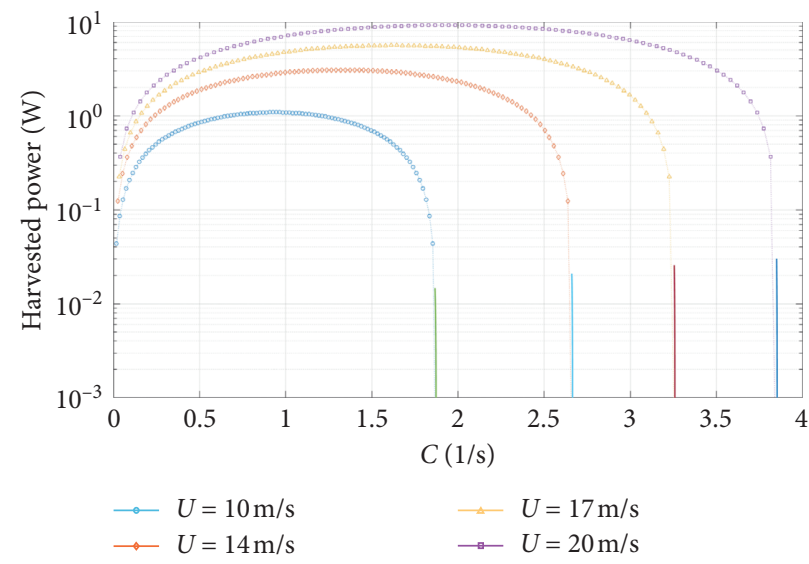

Figure 4: Analytical solutions (dash lines), numerical solutions (symbol), and corrected solutions (lines) of the amplitudes of the harvested power versus the parameter $C$ and free stream velocity.

TAble 1: Regular circuits.

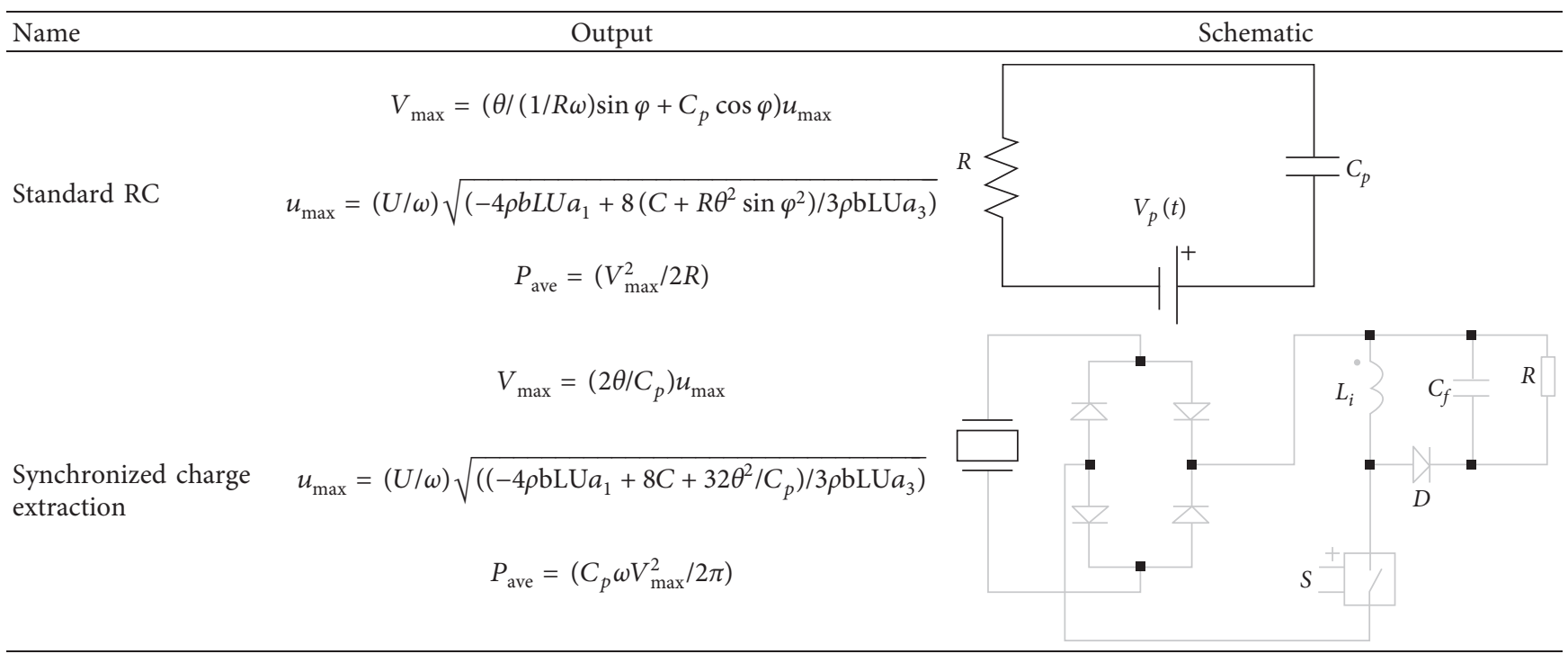




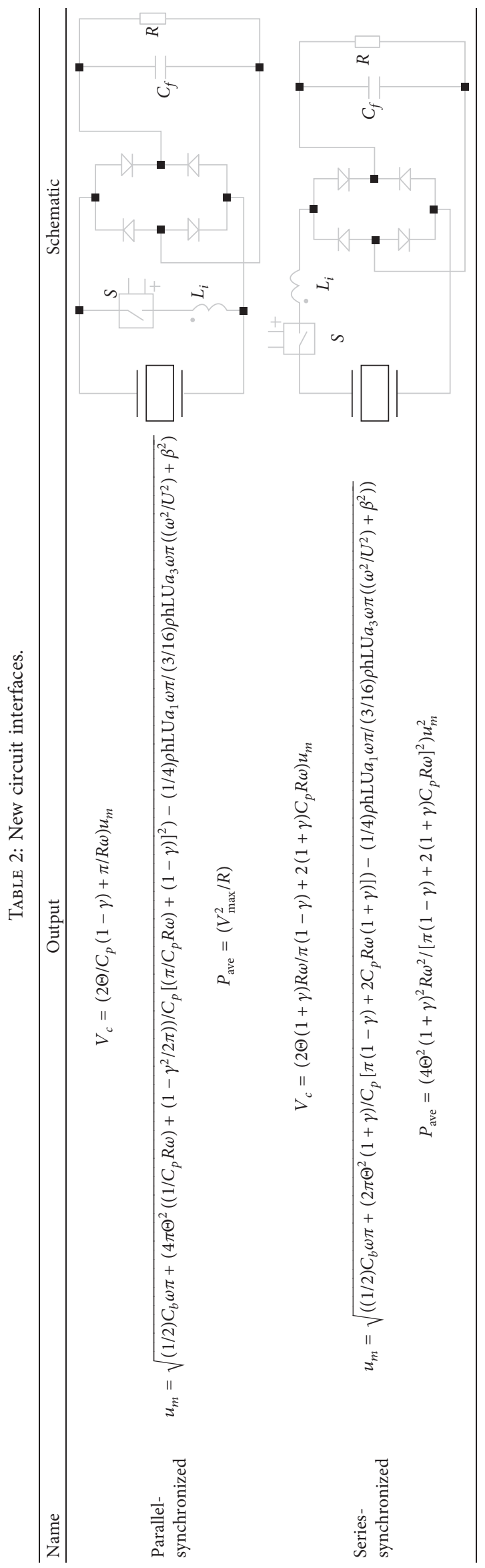




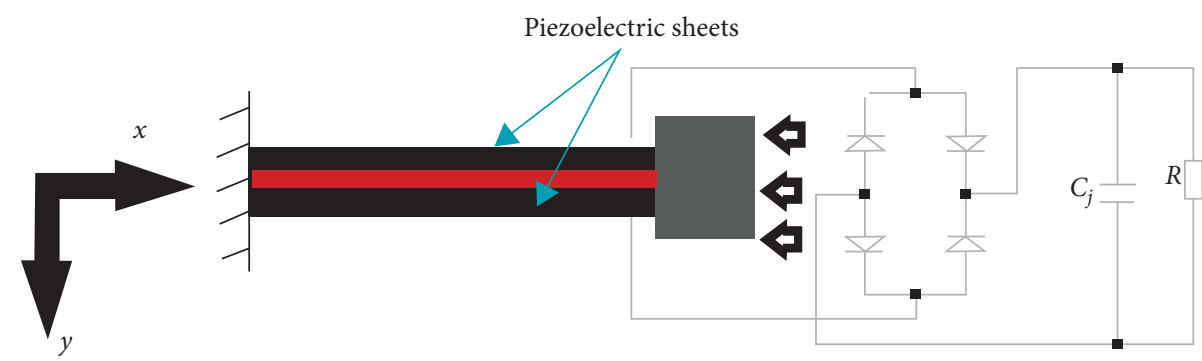

FIgURE 5: Schematic of the galloping piezoelectric energy harvester for the case of wind direction parallel to the cantilever beam.

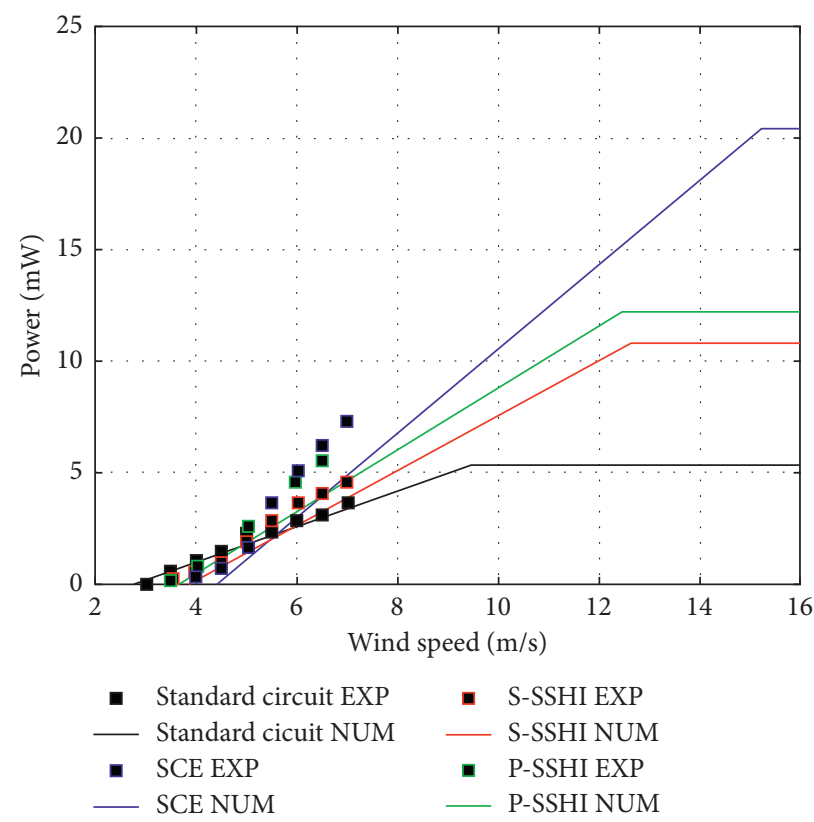

Figure 6: Maximum obtainable various galloping piezoelectric energy harvester for the case of wind direction parallel to the cantilever beam.

$16.1 \mathrm{~m} / \mathrm{s})$, the optimal values of the $C$ $\left((\partial C / \partial R)=0 \Longrightarrow C_{o}=\left(\theta_{p}^{2} / 2 C_{p} \Omega\right)\right)$ is found by

$$
\begin{aligned}
R_{o} & =\left(C_{p} \Omega\right)^{-1}, \\
U_{o}^{\text {onset }} & =\frac{4 \xi \omega+\theta_{p}^{2}}{C_{p} \Omega \rho_{\text {air }} b_{\text {tip }} a_{1}} \frac{1}{\phi^{2}(L) L_{\text {tip }}+\phi(L) \phi^{\prime}(L) L_{\text {tip }}^{2}+1 / 3 \phi^{\prime 2}(L) L_{\text {tip }}^{3}} \\
A_{\max , o} & =\sqrt{\frac{\left(\theta_{p}^{2} /\left(2 C_{p} \Omega\right)\right)+2 \xi \omega-A}{0.75 B \Omega^{2}}} \phi(L), \\
P_{\max , o} & =\frac{\left(\theta_{p}^{2} /\left(2 C_{p} \Omega\right)\right)+2 \xi \omega-A}{1.5 B C_{p} \Omega} \\
\Omega & =\sqrt{\frac{\omega^{2}+\frac{\theta_{p}^{2}}{2 C_{p}} .}{\omega_{p}}}
\end{aligned}
$$


TABLE 3: Nomenclature.

\begin{tabular}{|c|c|}
\hline Symbol & Description \\
\hline$\vec{A}$ & $\left(\rho_{\text {air }} \mathrm{Ub}_{\text {tip }} a_{1} / 2\right)\left(\phi^{2}(L) L_{\text {tip }}+\phi(L) \phi^{\prime}(L) L_{\text {tip }}^{2}+\frac{1}{3} \phi^{\prime 2}(L) L_{\text {tip }}^{3}\right)$ \\
\hline$B$ & $\left(\rho_{\text {air }} b_{\text {tip }} a_{3} / 2 U\right)\left(\phi(L) \int_{0}^{L_{\text {tip }}} \phi(L)+s(\phi(L))^{3} \mathrm{~d} s+\phi(L) \int_{0}^{L_{\text {tip }}} s(\phi(L)) \int_{0}^{L_{\text {tip }}^{2}} s \phi^{\prime}(L)^{3}+\mathrm{d} s\right)$ \\
\hline$C$ & $\left(R \theta_{p}^{2} /\left(1+C_{p}^{2} \Omega^{2} R^{2}\right)\right)$ \\
\hline$C_{b}$ & $2 \zeta \omega_{n} / \phi^{2}\left(L_{t}\right)$ \\
\hline$M$ & $1 / \phi^{2}\left(L_{t}\right)$ \\
\hline$K$ & $\omega_{n}^{2} / \phi^{2}\left(L_{t}\right)$ \\
\hline$\Theta$ & $\chi / \phi\left(L_{t}\right)$ \\
\hline$\rho_{\text {air }}$ & Air density \\
\hline$L$ & Length of the beam \\
\hline$F_{\text {tip }}$ & $\left(\rho_{\text {air }} U^{2} b_{\text {tip }} / 2\right) \int_{0}^{L_{\text {tip }}} a_{1}\left(\dot{y}_{L}+s \dot{y}_{L}^{\prime} / U\right)+a_{3}\left(\dot{y}_{L}+s \dot{y}_{L}^{\prime} / U\right)^{3} \mathrm{~d} s$ \\
\hline$M_{\text {tip }}$ & $\left(\rho_{\text {air }} U^{2} b_{\text {tip }} / 2\right) \int_{0}^{L_{\text {tip }}} a_{1}\left(\dot{y}_{L}+s \dot{y}_{L}^{\prime} / U\right)+a_{3}\left(\dot{y}_{L}+s \dot{y}_{L}^{\prime} / U\right)^{3} s \mathrm{~d} s$ \\
\hline$M_{\text {base }}$ & $F_{\text {tip }} L+M_{\text {tip }}$ \\
\hline$b_{\text {tip }}$ & Width of the tip body \\
\hline$L_{\text {tip }}$ & Length of the tip body \\
\hline$a_{1}, a_{3}$ & Aerodynamic force coefficients \\
\hline$R$ & Load resistance \\
\hline$Q$ & Quality factor \\
\hline$\xi$ & Damping ratio of the structure \\
\hline$\phi$ & Mode shape of the structure \\
\hline$\theta_{p}$ & Electromechanical coefficient of piezoelectric material \\
\hline$\sigma_{Y}$ & Yield strength of piezoelectric material \\
\hline$C_{p}$ & Capacity of piezoelectric layer \\
\hline$U^{p}$ & Wind velocity \\
\hline$V$ & Piezoelectric voltage \\
\hline$\beta$ & $\left(\phi^{\prime}\left(L_{t}\right) / \phi\left(L_{t}\right)\right)$ \\
\hline$\Omega$ & Angular velocity of the motion \\
\hline$\chi$ & First natural angular velocity of the cantilever beam \\
\hline$\omega$ & $e^{-\pi / 2 Q}$ \\
\hline$\gamma$ & Cantilever-beam displacement \\
\hline
\end{tabular}

As for the current parameters, this value of the $C$ parameter is less than the allowed values of $C$ parameter; for harvested power, the discussion is not necessary.

Analytical solutions for galloping-based piezoelectric energy harvesters with various interfacing circuits are summarized in Table 1. By assuming $V=V_{m} \cos (\omega \mathrm{t}+\varphi)$ and $u=u_{m} \cos (\omega \mathrm{t})$ where $\tan \varphi=\left(1 / \mathrm{RC}_{p} \omega\right)$, the maximum obtainable average power in a standard $\mathrm{RC}$ circuit as a function of deflection limit is

$$
P_{\max }=\frac{R}{2}\left(\omega \theta \sin \varphi \delta_{\max }\right)^{2},
$$

and for the synchronized charge extraction,

$$
P_{\text {max }}=\frac{2 \omega}{\pi C_{p}}\left(\theta \delta_{\max }\right)^{2} \text {. }
$$

For the case of wind direction parallel to the cantilever beam (see Figure 5), the governing equations are

$$
\begin{aligned}
& M \ddot{u}(t)+C_{b} \dot{u}(t)+K u(t)+\Theta V(t)=\frac{1}{2} \rho h L U^{2}\left[a_{1}\left(\frac{\dot{u}(t)}{U}+\beta u(t)\right)+a_{3}\left(\frac{\dot{u}(t)}{U}+\beta u(t)\right)^{3}\right], \\
& I+C_{p} \dot{V}-\Theta \dot{u}=0 .
\end{aligned}
$$

Various circuit interfaces are shown in Table 2. Maximum obtainable various galloping piezoelectric energy harvester for the case of wind direction parallel to the cantilever beam is plotted in Figure 6. The beam data is obtained from the energy harvester 2 in Table 3 of Zhao and Yang [8].

\section{Conclusion}

In this study, the nonlinear model of the galloping cantilever beam used for piezoelectric energy harvesting is simulated numerically with respect to the failure criteria as a limit of the maximum obtainable power. The ideal case of such 
system is compared with the case of maximum stress limited due to the yielding stress of piezoelectric material. The results show that the mechanical limits of the system do not allow us to obtain the anticipated values in theory, and the feasible values are 2-3 orders of magnitude lower than prediction values. Hence, the fracture limitation should be considered in the process of the design of galloping-based energy harvesters with piezoelectric materials. Furthermore, the current research proposes for engineering applications, and designing the control system for the amplitude of galloping is necessary as well. Finally, maximum obtainable average power in a standard RC circuit as a function of deflection limit and synchronized charge extraction is obtained. In addition, four electrical interfaces in gallopingbased energy harvesters are assessed. The results are for a feeble coupling SCE circuit, which is reasonable at higher wind while SSHI suits low wind speed. The standard circuit is suggested for strong electromechanical pairing, and the SCE has the best strength against the wind and can produce the highest value of power.

\section{Data Availability}

No data were used to support this study.

\section{Conflicts of Interest}

The authors declare that they have no conflicts of interest.

\section{References}

[1] A. Barrero-Gil, G. Alonso, and A. Sanz-Andres, "Energy harvesting from transverse galloping," Journal of Sound and Vibration, vol. 329, no. 14, pp. 2873-2883, 2010.

[2] J. Sirohi and R. Mahadik, "Piezoelectric wind energy harvester for low-power sensors," Journal of Intelligent Material Systems and Structures, vol. 22, no. 18, pp. 2215-2228, 2011.

[3] J. Sirohi and R. Mahadik, Journal of Vibration and Acoustics, vol. 134, Article ID 011009, 2012.

[4] A. Abdelkefi, Z. Yan, and M. Hajj, Smart Materials and Structures, vol. 22, 2013.

[5] T. Tan and Z. Yan, "Analytical solution and optimal design for galloping-based piezoelectric energy harvesters," Applied Physics Letters, vol. 109, no. 25, p. 253902, 2016.

[6] M. Y. A. Jamalabadi, K. M. Kwak, and S. J. Hwan, KSNVE, vol. 10, p. 54, 2016.

[7] M. Y. A. Jamalabadi, K. M. Kwak, and S. J. Hwan, KSNVE, ASK and KSME, vol. 4, p. 478, 2017.

[8] L. Zhao and Y. Yang, "Comparison of four electrical interfacing circuits in wind energy harvesting," Sensors and Actuators A: Physical, vol. 261, pp. 117-129, 2017. 